Energy Research Journal 1 (2): 68-72, 2010

ISSN 1949-0151

(C) 2010 Science Publications

\title{
Improving the Polypropylene-Clay Composite Using Carbon Nanotubes as Secondary Filler
}

\author{
${ }^{1}$ Salawudeen T. Olalekan, ${ }^{1}$ Suleyman A. Muyibi, ${ }^{2}$ Qasim H. Shah, ${ }^{1}$ Ma' an, F. Alkhatib, \\ ${ }^{1}$ Faridah Yusof and ${ }^{3}$ Isam Y. Qudsieh \\ ${ }^{1}$ Nanoscience and Nanotechnology Research Group, Department of Biotechnology Engineering, \\ Faculty of Engineering, International Islamic University Malaysia, \\ P.O. Box 10, 50728 Kuala Lumpur, Malaysia \\ ${ }^{2}$ Department of Mechanical Engineering, Faculty of Engineering, \\ International Islamic University Malaysia, Malaysia \\ ${ }^{3}$ Department of Chemical Engineering, Faculty of Engineering, \\ Jazan University, P.O. Box 706, Jazan 45142, Saudi Arabia
}

\begin{abstract}
Problem statement: Current researches have shown that the thermoplastic-clay nanocomposites have failed to live up to the earlier predictions albeit they have found some niche area of applications. This research work therefore aimed at studying the effect of carbon nanotube as secondary filler on the mechanical properties of polypropylene-clay nanocomposite. Approach: Hybrid polypropylene-clay nanocomposite was prepared in the presence of Multiwall Carbon Nanotubes (MWCNTs) as secondary additives using melt intercalation process. The effect of Multiwall Carbon Nanotubes (MWCNTs) on the polypropylene/clay matrix was investigated in terms of dispersion using XRD, tensile test (ASTM D 638) and notched Izod impact test (ASTM D256). These were compared with the conventional polypropylene-clay nanocomposite. Results: The resulting composite shows about $42 \%$ increase in the modulus, $26.20 \%$ in the tensile strength and $13.30 \mathrm{Kj} \mathrm{m}^{-2}$ impact strength when compared with binary combination of PP/Clay nanocomposite. XRD patterns of PP/Clay and PP/Clay/MWCNT nanocomposites show different diffraction peaks which are indications of intercalation mixed with macromixing. Conclusion: This study showed that MWCNT can successfully address the common shortcomings peculiar to PP/Clay nanocomposite.
\end{abstract}

Kew words: Carbon nanotubes, clay, MWCNTs, nanocomposite, thermoplastic, XRD

\section{INTRODUCTION}

Polymer reinforced with Carbon Nanotubes and Nanoclay are receiving research attention for various industrial applications such as automobile interior and exterior accessories, air and space craft, electronics, civil constructions and fabrications of process vessels (Atard et al., 2001). Researches on microcomposite have reached the limit of optimization in property enhancement because the achieved properties usually involved a lot of trade off (Pulickel et al., 2005). Stiffness is traded for toughness, or toughness is obtained at cost of optical clarity and sometimes, flexural strength is traded off for tensile property. To be précised, polymer nanocomposite is a better alternative to the conventional polymer composite made from microfillers in terms of strength, resistance to corrosion and thermal stability.
Dispersion of clays and clay minerals in polymer matrices usually results to a product (polymer/clay nanocomposite) that will combine the properties of both the polymer and the filler and hence material of an enhanced property is produced. These hybrids often lead to improved mechanical (Fornes et al., 2001), electrical insulation (Kato et al., 1997) and flame retardancy (Garcia, et al., 2003). The most essential factor that leads to optimal composite properties is good dispersion, (intercalation and exfoliation) and spatial distribution of the filler in the matrix (Maged et al., 2003).

Fillers are generally characterized based on their physical parameters like shape, size and their reinforcing effect when dispersed into a continuous polymer matrix. These physical parameters could be defined in terms of aspect ratio, packing fraction and

Corresponding Author: Salawudeen T. Olalekan, Nanoscience and Nanotechnology Research Group, Department of Biotechnology Engineering, Faculty of Engineering, International Islamic University Malaysia, P. O. Box 10, 50728 Kuala Lumpur, Malaysia 
surface area (Nello, 2005). The aspect ratio is described as a ratio of the major to the minor dimension and it defines the load transfer efficiency from the polymer matrix and the continuous phase to the filler. The packing fraction defines the volume occupied by solid particles over the total volume. It is correlated to the aspect ratio by an inverse relationship, that is, the higher the aspect ratio, the lower the packing fraction. This implies that the fillers exhibiting high reinforcing effect must have a low packing fraction or high aspect ratio (Salawudeen et al., 2008).

The mechanical property of carbon Nanotube is exciting, since they are considered as the ultimate carbon material ever made (Oliver and Micheal Claaes 2006). The most important application of carbonnanotubes, based on their mechanical strength is as reinforcements in composite materials (Pulickel et al., 2005). Compared with clay and other organic fibres which usually lack one or more of these properties, carbon nanotubes show a unique combination of stiffness, strength and tenacity (Dean et al., 2007). Also in comparison to the conventional organic and inorganic fillers, Carbon nanotube has therapeutic properties and hence can address the most common drawbacks peculiar to the conventional binary composites at minimal loading. For example carbon nanotubes is soft and elastic in the radial direction and hence can favorably address the problems of poor elongation and flexural strength found in most conventional binary composites (Andreas and Walter, 2007) and due to its high young modulus and tensile strength, it has a better potential for reinforcement than any other known nanomateria.

Due to the problem of agglomeration that usually resulted to insufficient load transfer between the polymer matrix and the nanofillers, multiwall carbon nanotubes is always the carbon nanotube of choice in the carbon nanotubes/polymer nanocomposite because it has a good adhesion with polymer matrix (Vaia et al., 1993). Nanomaterials have been dispersed in a variety of polymer matrices and different shortcomings were reported (Micheal and Connel, 2006). No attempt has been made to produce a hybrid that will combine two fillers in a matrix. In this study, an attempt was made to compare the mechanical properties of the conventional binary nanocomposite to a ternary nanocomposite which combines carbon nanotubes and nanoclay as reinforcement in polypropylene matrix. Such products will address most common shortcomings like low mechanical properties and high production cost which normally resorted when only CNT is used as filler.

\section{MATERIALS AND METHODS}

Materials: Polypropylene homopolymer was supplied by Medigene Sdn. Bhd Malaysia. Carbon nanotubes with outer diameter ranging between 10 and $20 \mathrm{~nm}$ and percentage purity $>95 \%$ was supplied by (Bahad SDN, Malaysia). Modified clay was prepared in Biochemical Laboratory in the Department of Biotechnology Engineering, IIUM, Malaysia using bentonite clay manufactured by Acros organics New Jersey, USA). Maleic Anhydride grafted Polypropylene (MAgPP) and Octadecylamine (90\%) were supplied by Suka Chemicals Malaysia. All the above materials apart from bentonite clay were used without any further treatment.

Clay modification: About $1000 \mathrm{~mL}$ of distilled water was measured into $2 \mathrm{~L}$ capacity beaker, heated and maintained at a temperature of $80^{\circ} \mathrm{C}$ in a water bath. Twenty gram of clay sample was measured into the hot water and allowed to disperse under mixer rotating at (300 rpm) for $1 \mathrm{~h}$. A solution containing $7.5 \mathrm{~g}$ octadecylamine with $4.2 \mathrm{~mL}$ of concentrated hydrochloric acid $(\mathrm{HCl})$ in $500 \mathrm{ml}$ hot distilled water maintained at $80^{\circ} \mathrm{C}$ was subsequently added into the mixture and allowed to mix for another $1 \mathrm{hr}$ with the speed adjusted to $400 \mathrm{rpm}$. After this, the solid was filtered and washed severally with $1000 \mathrm{ml}$ hot distilled water using vacuum filtration apparatus. The organoclay produced was dried in an oven maintained at $60^{\circ} \mathrm{C}$ for $36 \mathrm{~h}$.

Preparation of polypropylene nanocomposites: Melt mixing method has been reported to be a suitable method for the production of polypropylene nanocomposites due to its environmental friendliness (Quang and Donald, 2007) and ability to achieve better dispersion at low cost (Quang and Donald, 2007) because no additional solvent is required. Melt intercalation therefore was adopted in this work.

Preparation of polypropylene/clay nanocomposite: Preparation of Polypropylene/clay was effected by direct melt mixing of PP, MAgPP at predetermined ratio in a Hakee mixer type (Rheomix 600P) for $3 \mathrm{~min}$ before the introduction of modified clay through the hopper. The melt mixing conditions were $180^{\circ} \mathrm{C}$ and at a rotor speed of $100 \mathrm{rpm}$. The entire mixture was allowed to mix for 10minutes before the nanocomposite was unloaded and manually pelletized. The clay percentage was varied between 1.0 and $6.0 \mathrm{wt} \%$ of polypropylene. 
The nanocomposite pellets were dried at $80^{\circ} \mathrm{C}$ in a vacuum oven for $12 \mathrm{~h}$ before injection molded using injection molding machine of type (HM 600/210). The composites were molded into a standard dumb bell shape using injection molding machine at melt temperature of $195^{\circ} \mathrm{C}$ and a mold temperature of $180^{\circ} \mathrm{C}$. The mechanical properties of the injection plaques were determined. The formulation of the plaque with best mechanical properties was used in the preparation of the master batch. This master batch was used as intermediate raw material for the production of ternary nanocomposite. The progress in the mixer was monitored using the plot shown in Fig. 1.

Preparation of PP/MNC/MWCNTs: Ternary nanocomposite production was effected by first preparing the $\mathrm{PP} /$ Clay composite using the formulation that gave the best mechanical properties as described above. PP/Clay pellet was melted in the hot mixer before the introduction of Multiwall Carbon Nanotubes in the prescribed quantity through hopper at $185^{\circ} \mathrm{C}$ melting temperature. The entire mixture was allowed to mix for a period of 10 min before homogeneity was attained. The progress in the mixer was monitored using the same plot shown in the Fig. 1. The nanocomposite was manually pelletized and injection molded into the standard shape. The injection molding operating conditions were kept at a melt temperature of $195^{\circ} \mathrm{C}$ and a mold temperature of $180^{\circ}$ at 700 bar injection pressure.

Mechanical test: Tensile elongation and tensile modulus measurements are among the most important indications of strength in a material and are the most widely specified properties of plastic materials (Myer, 2002).

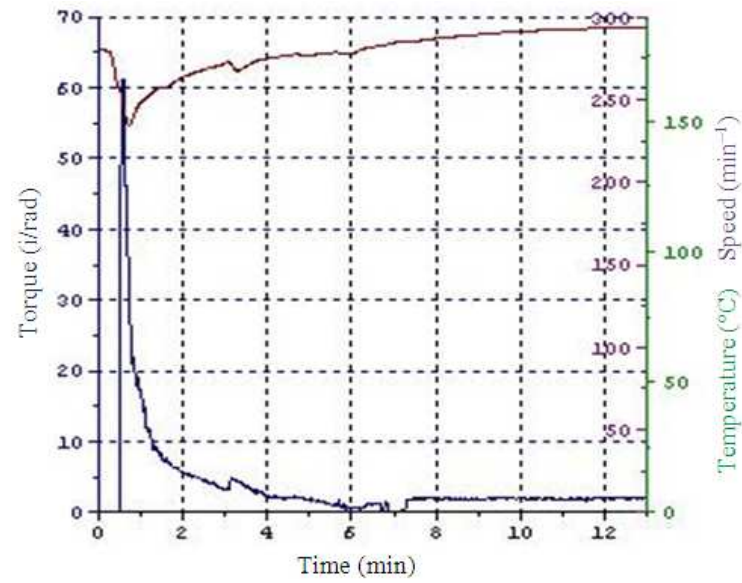

Fig. 1: Plot of torque, speed and temp versus time
The tensile test was carried out using (ASTM D638) on the molded plaques using Universal Tensile Testing Machine (Lloyd Instrument model LR10K). The test specimen was inserted into the self aligning grips employed for holding the test specimen between the fixed member and the movable member. The test specimen was deformed at various strain rates up to post-yielding and the stress strain and force displacement curves were automatically plotted on the system attached to the equipment. From the curves, the Tensile strength and the young modulus were determined. Similarly, the impact test was carried out using advance pendulum impact (Dynisco polymer test model API-230-0). The rectangular bars of the injection molding plaques were notched prior to the test using sample Notcher, dynisco polymer test (model ASN230-M).

Determination of degree of dispersion: The degree of dispersion was determined by Shimadzu Xray Diffractometer (LabXRD-6000). Nanocomposite sample was prepared following the specifications and mounted on the sample holder of XRD. Having ascertained that all the recommended precautions were already in place, the diffraction was conducted at $2 \theta=70^{\circ}$. The peaks were plotted and interpreted.

\section{RESULTS}

Figure 2 shows the tensile properties (tensile strength and elastic modulus) of polypropylene-clay nanocomposte. This was used to bench marked the properties of ternary nanocomposite.

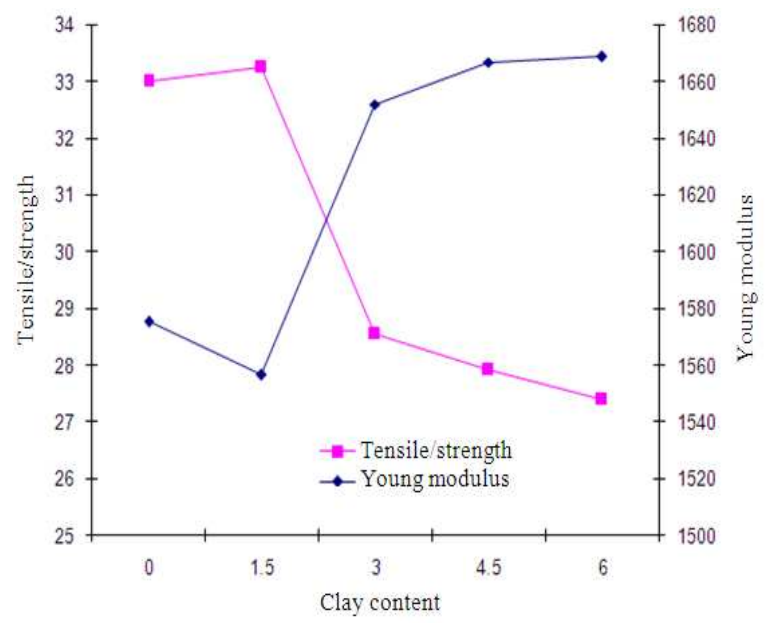

Fig. 2: Effect of clay content on the mechanical properties of PP-clay nanocomposite 
Energy Rec. J. 1 (2): 68-72, 2010

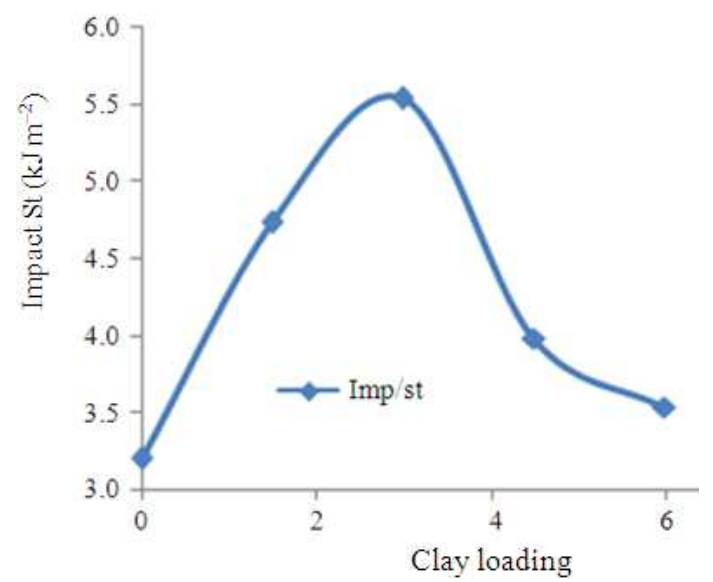

Fig. 3: Effect of Clay Content on Impact the impact strength of polypropylene nanocomposite

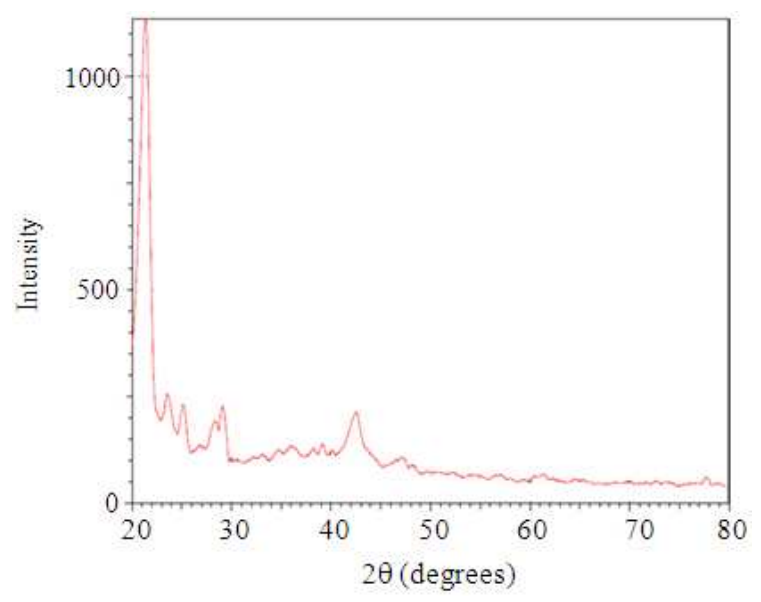

Fig. 4: XRD curves of PP/clay nanocomposite

Table 1: Comparison between binary and ternary Nanocomposite

\begin{tabular}{llll}
\hline & \multicolumn{3}{c}{ Mechanical properties } \\
Composite & Ten. St (MPa) & Yong mod (MPa) & Imp. St $\left(\mathrm{kJ} \mathrm{m}^{-2}\right)$ \\
\hline PP/clay & 28.55 & 1588.27 & 5.55 \\
PP/clay/CNTs & 36.13 & 2250.00 & 6.28 \\
\hline
\end{tabular}

The effect of impact strength studied over a wide range of clay loading on polypropylene is shown in Fig. 3. Similarly, Table 1 shows the difference between the mechanical properties of the master batch (pp/clay) and the ternary nanocomposites after the addition of multiwall carbon nanotube.

The X-ray diffraction patterns of both nanocomposites were shown in Fig. 3 and 4. These were determined over the same diffraction angle for basic comparison.

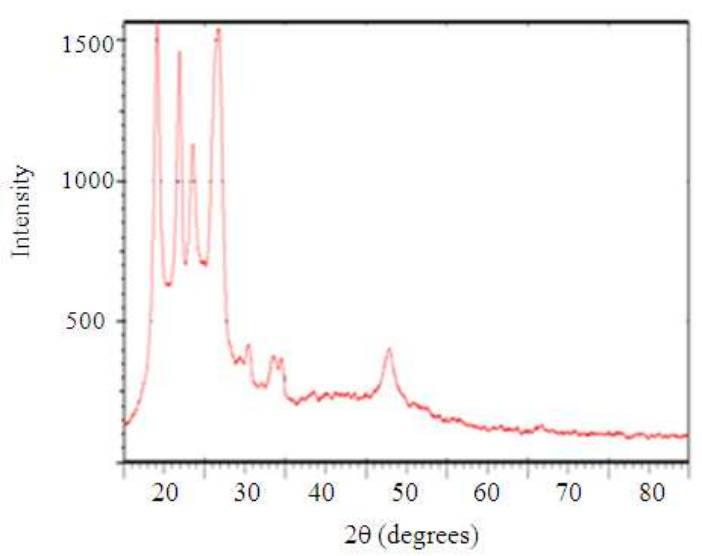

Fig. 5: XRD curves of PP/clay/MWCNT nanocomposite

\section{DISCUSSION}

The mechanical properties of the binary nanocomposites were evaluated by tensile and impact testing (Fig. 2 and 3). As shown in Fig. 2. PP/Clay system shows a slight decrease in Young's modulus at a low filler loading. This is characteristic of Maleic anhydride grafted polypropylene which was added to promote PP/Clay intercalation due to the apola nature of polypropylene. When the loading of the modified clay was above $2 \mathrm{wt} \%$ a reasonable increase in stiffness (characterized by the Young's modulus) was observed as filler loading increases. The ultimate Young's modulus lies between 1652.27 and $1666.87 \mathrm{MPa}$ which corresponds to point 3 and 4.5 in the plot.

The behavior of the Tensile strength with respect to clay content was in the reverse order to that of Young's modulus as shown in Fig. 2. But the rate of decrease in the strength reduces between point 3 and 4.5. This justified the claimed that the ultimate may lies between 3 and $4.5 \mathrm{wt} \%$.

To further strengthen the results obtained in terms of Tensile strength and Young's modulus, the impact property of the plague was tested using Notched Izod Impact Test (ASTM D256). Figure 3 shows a remarkable increase in impact strength as clay contents increases and attained an optimum at $3 \%$ by weight of clay. This is because augmenting the clay content above the ultimate value resulting to agglomeration which tends to reduce some properties in the polypropylene nanocomposite.

Judging from the above results, the ultimate clay content was taken as 3\% and this was used in the preparation of master batch (PP/Clay) which was later used in the preparation of ternary nano composite (PP/Clay/MCNTs). 
When the MWCNT was added to the PP/Clay nanocomposite, the comparison was as shown in Table 1. Both the tensile strength and the elastic modulus experienced an appreciable increment of $42 \%$ and $26.2 \%$ respectively while the impact strength gained an increase of $13.3 \% \mathrm{Kj} \mathrm{m}^{-2}$. This behavior justified the potential properties of carbon nanotube.

The XRD patterns of both the pp/clay and the ternary nanocomposites shown in Fig. 4 and 5 depicted that PP/Clay has better dispersion when compared with PP/Clay/MWCNT. This is because the $\mathrm{X}$ ray pattern shows a much more defined peak in the PP/Clay/MWCNT than in PP/Clay. This implies that MWCNTs were not well dispersed in the composite and thus lead to the formation of agglomerates (characteristics of nanomaterials) in the system.

\section{CONCLUSION}

In this study multiwall carbon nanotubes have been used to improve mechanical performance of polypropylene/Clay nanocomposite due to its higher aspect ratio and surface area which facilitates its better dispersion in the matrix. However, the XRD analysis showed the combination of both intercalation and micromixing due to some agglomeration formed during the process.

\section{ACKNOWLEDGEMENT}

The authors are grateful to the Malaysian Ministry of Higher Education for funding this project under the Fundamental Research Grant Scheme project no. FRGS 0206-56.

\section{REFERENCES}

Atard, G.S., S.A.A. Leclerc, S. Maniguest, A.E. Russel and I. Nandha-kumarl., 2001. Liquid crystal phase templated mesoporous platinum alloy. Microporous Mesoporous Mater., 44: 159-163. DOI: $10.1016 /$ SI387-1811(01)00180-9

Andreas, F. and K. Walter, 2007. Polypropylene carbon nanotubes by in situ polymerization. Composite Sci. Technol. 67: 906-915. DOI: 10.1016/j.compscitech.2006.01.034

Dean, S., Y. Wai, K.Y. Robert, K. Zhuo and Y. Jinghua, 2007. An investigation on the dispersion of montmorillonite (MMT) primary particles in PP matrix. Eur. Polymer J., 43: 3250-3257. DOI: 10.1016/j.eurpolyj.2007.05.030
Fornes, T.D., P.J. Yoon, H. Keskkula and D.R. Paul, 2001. Nilon 6 nanocomposite: The effect matrix molecular weight. Polymer, 42: 9929-9940. DOI: 10.1016/S0032-3861(01)00552-3

Garcia, D., O. Picazo, J.C. Merino and J.M. Pasyor, 2003. Polypropylene clay nanocomposite: Effect of compatibilizing agents on clay dispersion. Eur. Polymer J., 39: 945-950. DOI: 10.1016/S00143057(02)00333-6

Kato, M., A. Usuki and A. Okada, 1997. Synthesis of polypropylene oligomer-clay intercalation compounds. J. Applied Polymer Sci., 66: 1781-1785. DOI: $\quad 10.1002 /(S T C I) 1097-$ 4628(19971128)66:9<1781::AID-PP17>3.0C0;2-Y

Maged, A., P. Michael and W. Ulrich, 2003. Surface treatment of clay minerals-thermal stability, basalplane spacing and surface coverage. J. Mater. Chem., 13: 2359-2366. DOI: b302331a.

Micheal, J. and O. Connel, 2006. Carbon nanotubes Properties and Applications. 1st Edn., Taylor and Francis group Boca Roton, London, New York, IABN: 10:0-8493-2748-2, pp: 214-255.

Myer, K., 2002. Handbook of Materials Sellection. 1st Edn., John Willey and Sons, Inc. New York, ISBN: 0-471-35924-6.

Nello, P., 2005. Polypropylene Hand Book. 2nd Edn., Germany: Hanser Gadner Publication, Inc., ISBN: 0-8247-4064-5, pp: 326.

Oliver, D. and Micheal Claaes, 2006. Carbon nanotubes: Reviewing their multifunctional properties for polymers. Proceeding of the 2nd International Conference of Filler for Polymers pp: 1-10. ISBN: 1859575609.

Pulickel M.A,, S.S. Lida and V.B. Paul, 2005. Nanocomposite Science and Technology. 1st Edn., Willey-VCH Verlay $\mathrm{GmbH}$ and Co. K. Ga Weinheim, ISBN: 3-527-30359-6, pp: 31.

Quang, T.N. and G.B. Donald, 2007. An improve technique for exfoliating and dispersing nanoclay particles into polymer matrices using supercritical carbon dioxide. Polymer, 4: 6923-6933. DOI: 10.1016/j.polymer.2007.09.015

Salawudeen, T.O, Y.Q. Isam, F.A. Maan, F.A. Maan and A.M. Suleyma, 2008. Potential application of carbon nanotubes as filler element in the production of polymer nanocomposites: A Review. Proceeding of the Regional Symposium on Chemical Engineering in Conjunction with Symposium of Malaysian Chemical Engineers, pp: 239-243. ISBN: 978-983-2982-23-4.

Vaia, R.A., H. Ishii and E.P. Giannelis, 1993. Synthesis and properties of two-dimensional nanostructure by direct intercalation of polymer melt in layered silicates. Chem. Mater., 5: 1994-1993. DOI: $10.1021 / \mathrm{cm} 00036 \mathrm{a} 004$ 